\title{
Molecular characterization of SQUAMOSA PROMOTER BINDING PROTEIN-LIKE (SPL) gene family from Citrus and the effect of fruit load on their expression
}

\author{
Liron Shalom ${ }^{1,2}$, Lyudmila Shlizerman ${ }^{1}$, Naftali Zur ${ }^{1}$, Adi Doron-Faigenboim ${ }^{1}$, \\ Eduardo Blumwald ${ }^{3}$ and Avi Sadka ${ }^{1 *}$ \\ ${ }^{1}$ Department of Fruit Tree Sciences, Agricultural Research Organization, The Volcani Center, Bet Dagan, Israel, ${ }^{2}$ The Robert \\ H. Smith Faculty of Agriculture, Food and Environment, The Hebrew University of Jerusalem, Rehovot, Israel, ${ }^{3}$ Department of \\ Plant Sciences, University of California, Davis, Davis, CA, USA
}

Edited by:

Anna Maria Mastrangelo,

CRA-Centro di Ricerca per la

Cerealicoltura, Italy

Reviewed by:

Cai-Zhong Jiang,

United States Department of Agriculture - Agricultural Research

Service, USA

Concetta Licciardello, Consiglio per la Ricerca in Agricoltura

e l'economia Agraria, Italy

*Correspondence:

Avi Sadka,

Department of Fruit Tree Sciences, Agricultural Research Organization,

The Volcani Center, PO Box 6, Bet

Dagan, Israel

vhasadka@volcani.agri.gov.il

Specialty section:

This article was submitted to

Crop Science and Horticulture, a section of the journal

Frontiers in Plant Science

Received: 24 March 2015 Accepted: 15 May 2015

Published: 27 May 2015

Citation:

Shalom L, Shlizerman L, Zur N, Doron-Faigenboim A, Blumwald E and

Sadka A (2015) Molecular

characterization of SQUAMOSA PROMOTER BINDING PROTEIN-LIKE (SPL) gene family from Citrus and the effect of fruit load on their expression.

Front. Plant Sci. 6:389.

do: 10.3389/fpls.2015.00389
We recently identified a Citrus gene encoding SQUAMOSA PROMOTER BINDING PROTEIN-LIKE (SPL) transcription factor that contained a sequence complementary to miR156. Genes of the SPL family are known to play a role in flowering regulation and phase transition. In Citrus, the mRNA levels of the gene were significantly altered by fruit load in buds; under heavy fruit load (ON-Crop trees), known to suppress next year flowering, the mRNA levels were down-regulated, while fruit removal (de-fruiting), inducing next-year flowering, resulted in its up-regulation. In the current work, we set on to study the function of the gene. We showed that the Citrus SPL was able promote flowering independently of photoperiod in Arabidopsis, while miR156 repressed its flowering-promoting activity. In order to find out if fruit load affected the expression of additional genes of the SPL family, we identified and classified all SPL members in the Citrus genome, and studied their seasonal expression patterns in buds and leaves, and in response to de-fruiting. Results showed that two additional SPL-like genes and miR172, known to be induced by SPLs in Arabidopsis, were altered by fruit load. The relationships between these factors in relation to the fruit-load effect on Citrus flowering are discussed.

Keywords: alternate bearing, Citrus, flowering, miR156, miR172, SPL

\section{Introduction}

During their growth, plants undergo a series of developmental transitions, regulated by a complex network of molecular factors that are activated and interact in response to endogenous and environmental cues. Research of juvenile-to-adult and vegetative-to-reproductive phase transitions in model and crop plants has revealed the importance of two microRNAs, miR156 and miR172, which coordinate these processes in an opposite manner: miR156 is highly abundant during the juvenile phase and gradually decreases with age; its overexpression prolongs the expression of juvenile features and significantly delays flowering (Wu and Poethig, 2006; Chuck et al., 2007); in contrast, $m i R 172$ abundance increases as the plant ages and its overexpression accelerates flowering (Aukerman and Sakai, 2003; Chen, 2004; Lauter et al., 2005; Wu and Poethig, 2006; Jung et al., 2007). In Arabidopsis, miR156 targets 10 out of 16 members of the SQUAMOSA PROMOTER 
BINDING PROTEIN-LIKE (SPL) family of transcription factors (TFs), which are characterized by a 76-amino acid DNAbinding domain named SBP. SPLs influence flowering in a number of ways. In the leaf, they promote flowering upstream of FLOWERING LOCUS T (FT) by up-regulating miR172, a repressor of APETALA2 (AP2)-like TFs which inhibits FT transcription (Mathieu et al., 2009). In the apex, the expression of several SPLs increases during early stages of flowering transition (Wang et al., 2009) and further activates important MADS-box genes by directly binding their promoters (Wang et al., 2009; Yamaguchi et al., 2009). In addition, flowering via the gibberellin (GA) pathway is mediated by SPLs (Yu et al., 2012), whereas the miR156-SPL module directly regulates $F T$ expression to control ambient temperature-responsive flowering (Kim et al., 2012). SPLs have also been identified as potential targets of the signaling molecule trehalose-6-phosphate, which positively regulates flowering in Arabidopsis (Wahl et al., 2013), suggesting a relationship between SPLs and the carbohydrate status of the plant. Aside from their regulatory role in floral transition, SPLs have been found to influence diverse physiological processes, such as sporogenesis (Unte et al., 2003), leaf development (Usami et al., 2009), copper homeostasis (Yamasaki et al., 2009), male fertility (Xing et al., 2010), gynoecium patterning (Xing et al., 2013), trichome development (Yu et al., 2010) and anthocyanin biosynthesis (Gou et al., 2011). The involvement of miR156, miR172, and SPLs in the juvenile-to-adult phase transition has also been demonstrated in woody perennials, including English ivy, eucalyptus, poplar, acacia and oak, in a manner similar to that in annual plants (Wang et al., 2011).

In addition to the age-dependent phase transition, perennials undergo seasonal phase transitions into flowering, which usually occur on an annual basis. However, in some tree species, flowering occurs biennially or even every few years. In biennial bearers, heavy fruit load 1 year (ON-Crop year) inhibits flowering the following year (OFF-Crop year), a phenomenon known as alternate bearing $(\mathrm{AB})$ (Monselise and Goldschmidt, 1982). Complete fruit removal (de-fruiting) from ON-Crop trees induces next-year flowering (return bloom). In a search for regulatory and other processes which are altered in the buds as a result of contrasting fruit loads in Citrus, we previously identified an SPL-like gene whose mRNA level was relatively high during OFF-Crop years and low during ON-Crop years (Shalom et al., 2012). Moreover, de-fruiting significantly induced this gene's expression in the bud within a short time (Shalom et al., 2014). Although this SPL-like gene contained a miR156binding site, no changes in $m i R 156$ levels were detected between buds of ON- and OFF-Crop trees, suggesting its regulation by other factors as well.

To the best of our knowledge, the above studies were the first to demonstrate a correlation between $S P L$-like gene expression and fruit load in fruit trees. However, further analyses are required to strength these relationships. In the current work, we performed a functional analysis of the Citrus SPL in Arabidopsis. The results showed it was able to promote flowering, while miR156 repressed its action. As in Arabidopsis, the Citrus genome contains additional $S P L$-like genes. Therefore, we also set out to identify all SPLs from Citrus and characterize their expression throughout the season and following de-fruiting. The responses of miR156 and miR172 to de-fruiting in Citrus buds are also characterized, and the relationships between them and SPLs with respect to $A B$ are discussed.

\section{Materials and Methods}

\section{Identification and Phylogeny of Citrus SPLs}

Citrus SPL genes were identified using the Citrus clementina genome (http://www.phytozome.net). Arabidopsis SPL sequences were compiled from the TAIR database (https://www.arabidopsis.org/). Alignments were performed by MUSCLE program using default parameters (http://www.ebi.ac. $\mathrm{uk} /$ Tools/msa/muscle/ (Edgar, 2004). A phylogenetic tree was constructed based on the maximum likelihood (ML) framework using Phyml software (Guindon and Gascuel, 2003) by the JTT matrix-based model. The tree was graphically designed with the use of FigTree version 1.4 (http://tree.bio.ed.ac.uk/software/ figtree/).

\section{Full-length Sequencing of CiSPL5 mRNA}

An Expressed Sequence Tags (EST) based SPL consensus sequence was pooled from the HarvEST Citrus database (http:// harvest.ucr.edu/) according to the probe set ID of the Citrus GeneChip Microarray (Affymetrix, Inc., Santa Clara, CA). Total RNA was extracted from buds of 15 -year-old Murcott mandarin (Citrus reticulata Blanco) trees grafted on sour orange (Citrus aurantium $\mathrm{L}$.), using the $\mathrm{CTAB}$ extraction method (Chang et al., 1993). RNA was treated with RQ1 RNase-free DNase (Promega, Fitchburg, WI) according to the manufacturer's instructions. $5^{\prime}$-RACE and 3'-RACE for CiSPL5 were carried out using the FirstChoice RLM RACE Kit (Ambion, Austin, TX). For 5' RACE, $10 \mu \mathrm{g}$ of total RNA was ligated to the RNA adapter after treatment with calf intestinal phosphatase and tobacco acid pyrophosphatase, followed by cDNA synthesis using random primers. For $3^{\prime}$-RACE, cDNA was synthesized using the oligo $\mathrm{d}(\mathrm{T})$ adapter supplied by the manufacturer. Outer PCR and inner PCR were carried out using the adapter primers, and primers specific for Citrus SPL (Supplementary Table 4). RACE products were gel-purified and cloned into pGEM T easy vector (Promega) for sequencing.

\section{Plasmid Construction and Production of Transgenic Plants}

The CiSPL5 transcript sequence (open reading frame [ORF] + $3^{\prime}$ untranslated region [UTR]) and a sequence lacking the $3^{\prime}$ UTR (ORF no $3^{\prime}$ UTR) were PCR-amplified with Pfu DNA polymerase (Thermo Fisher Scientific, Waltham, MA) using cDNA as the template. CiSPL5 ORF $+3^{\prime}$ UTR was amplified using the following primers: forward CTAAAGGAAAAGAC TGTCAAGGATT, reverse GCGTAACGATTGATTCCTCAG. CiSPL5 ORF no $3^{\prime}$ UTR was amplified using the following primers: forward CTAAAGGAAAAGACTGTCAAGGAT $\mathrm{T}$, reverse CTACTGAGGACCTACCCCTC. The sequence CiSPL5 ORF + mutated 3'UTR was generated by introducing 10 mutations into the predicted miR156-binding site using recombinant PCR. Primers used for recombinant PCR were: 
forward TCGCATATTCACTACTCTCTTCCTTAGGCTCCT CCTCT, reverse AGAGAGTAGTGAATATGCGACCTGCAA TGCAGAAAGTT in combination with the primers used for amplification of CiSPL5 ORF $+3^{\prime} \mathrm{UTR}$. All three constructs were cloned downstream of the CaMV $35 \mathrm{~S}$ promoter in pART27 using pART7 as an intermediate vector (Gleave, 1992). Arabidopsis plants (Columbia ecotype) were transformed using the floral dip method (Bechtold et al., 1993). Transformed plants were identified by kanamycin selection $(50 \mu \mathrm{g} / \mathrm{ml})$. Plants were grown under long-day ( $16 \mathrm{~h}$ light) or short-day ( $8 \mathrm{~h}$ light) conditions at $22^{\circ} \mathrm{C}$. Flowering time represents the appearance of the first open flower.

\section{Citrus SPLs Expression Analyses}

Complete fruit removal (de-fruiting) and sample collection were carried out as previously described (Shalom et al., 2014). For seasonal expression analyses, plant material was collected from a commercial orchard of 15-year-old Murcott mandarin (C. reticulata Blanco) trees grafted on sour orange (C. aurantium L.), located in the central coastal area of Israel, during the year 2014. Samples were collected from three biological replicates, each containing three OFF-Crop trees. Vegetative shoots, collected from the southeast side of the trees, were taken to the laboratory on ice. Buds and leaves were separated and immediately frozen in liquid nitrogen. Total RNA was extracted using the CTAB extraction method (Chang et al., 1993) and treated with RQ1 RNase-free DNase (Promega) according to the manufacturer's instructions. Primers for all of the identified Citrus SPLs were designed based on genomic sequences (Phytozome, http://www.phytozome.net/) using Primer3 software (Supplementary Table 1). To exclude putative antisense transcripts (ASTs) as templates in the realtime PCR, primers were designed to span exon-exon junctions or two different exons with a large intron between them. Real-time PCR was carried out as described (Shalom et al., 2012).

\section{miR156 and miR172 Analysis}

ctr-MIR156 and csi-MIR172a (one of several identified miR172 genes) were previously identified as Citrus microRNA genes transcribing precursors which generate mature miR156 and miR172 sequences, respectively (Song et al., 2009; Xu et al., 2010). Thus, measuring their expression may indicate the abundances of their compatible mature miRNAs. In the two available Citrus genome databases (Phytozome and http://citrus. hzau.edu.cn/orange/), both ctr-MIR156 and csi-MIR172a were not predicted as genes, probably duo to their relatively short lengths $(<200 \mathrm{bp})$ and the lack of sufficient open reading frames, and are therefore represented here by their names given upon identification. For the analyses of ctr-MIR156 and csi-MIR172a expression, Citrus microRNA (miRNA) precursor sequences (Supplementary Table 2) were compiled from the miRBase database (http://www.mirbase.org/). Abundance estimates were calculated for each sequence and sample using the RSEM software package (Li and Dewey, 2011) and Bowtie alignment program (Langmead et al., 2009). TMM (trimmed mean of $M$ values-weighted trimmed mean of the log expression values) normalization was performed using code in edgeR, as described by Robinson and Oshlack (2010), and applied to scale the FPKMvalues provided by the abundance estimation software (RSEM) across all samples (Haas et al., 2013). The abundance of mature miR156 and miR172a sequences was determined using TaqMan ${ }^{\circledR}$ Small RNA Assay Kits (Thermo Fisher scientific, Walthem, MA, USA) according to manufacturer's instructions; $10 \mathrm{ng}$ total RNA was used, and real-time PCR was run in a Rotor Gene $\mathrm{Q}$ instrument (Qiagen, Venlo, Netherlands). The results were normalized against the $\beta$-actin gene as described previously (Shalom et al., 2014).

\section{Statistical Analysis}

Real-Time PCR results were analyzed by One-Way analysis of variance (ANOVA) with Tukey-Kramer multiple comparison tests, as implemented in the software JMP version 10 (SAS Institute, Cary, NC, USA).

\section{Results}

\section{Gene Structure and Phylogeny of SPL Family Members from Citrus}

To identify Citrus SPL members the following approaches have been taken: (1) all sequences containing an SBP domain were compiled from the Citrus clementina genome database (http:// www.phytozome.net/), (2) proper identification of all matching sequences was confirmed by performing BLAST against two different Citrus genome databases (Phytozome and http://citrus. hzau.edu.cn/orange/) using the 16 SBP-domain sequences from Arabidopsis as queries, and (3) all SPL-related unigenes were pooled from the Citrus EST database (http://harvest.ucr.edu/) and BLASTed against the $C$. clementina genome database. Following removal of redundant sequences and alternative transcripts, a total of 15 SPL members were determined in the C. clementina genome (Figure 1). To date, only one mature miR156 sequence from Citrus has been experimentally validated (Xu et al., 2010). Of the 15 putative Citrus SPL transcripts, 10 contained sequences complementary to the 20-nucleotide mature miR156 sequence, with one or two mismatches at the 1st, 7th, or 9th nucleotide (Figure 1). Analysis of the other five SPL transcripts resulted in no significant matches. Alignment of the full-length protein sequences of Arabidopsis and Citrus showed no consensus sequences other than the SBP domain (not shown). Therefore, only the SBP domains (Supplementary Table 3) were used for the phylogenetic analysis (Figure 2). The results of this analysis suggested that gene multiplication occurred before separation of Citrus and Arabidopsis, which are considered to be taxonomically related. Only Ciclev10020532 contained an SBP domain that was somewhat unique to Citrus (Figure 2). In general, these SPLs could be classified into three subgroups. The first one (green clade) consisted of SPLs characterized by relatively short protein sequences ( $<200$ amino acids) and a miR156-binding site located within the $3^{\prime}$ UTR of the transcript. The Arabidopsis members of this group, AtSPL3, AtSPL4 and AtSPL5, showed the highest homology to three short Citrus SPL members: Ciclev10009879, Ciclev10017104 and Ciclev10016841 containing 


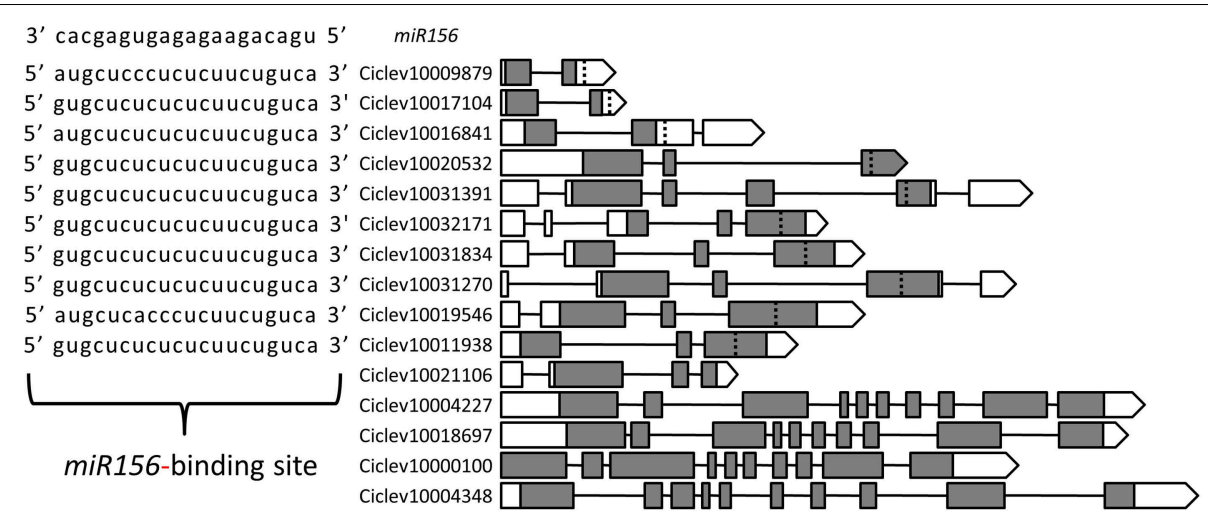

FIGURE 1|Genomic structure of Citrus SPL genes. Exon-intron organization of the primary transcript of each $S P L$ gene is based on the Citrus clementina genome (http://www. phytozome.net/). Exons are represented by boxes and introns by

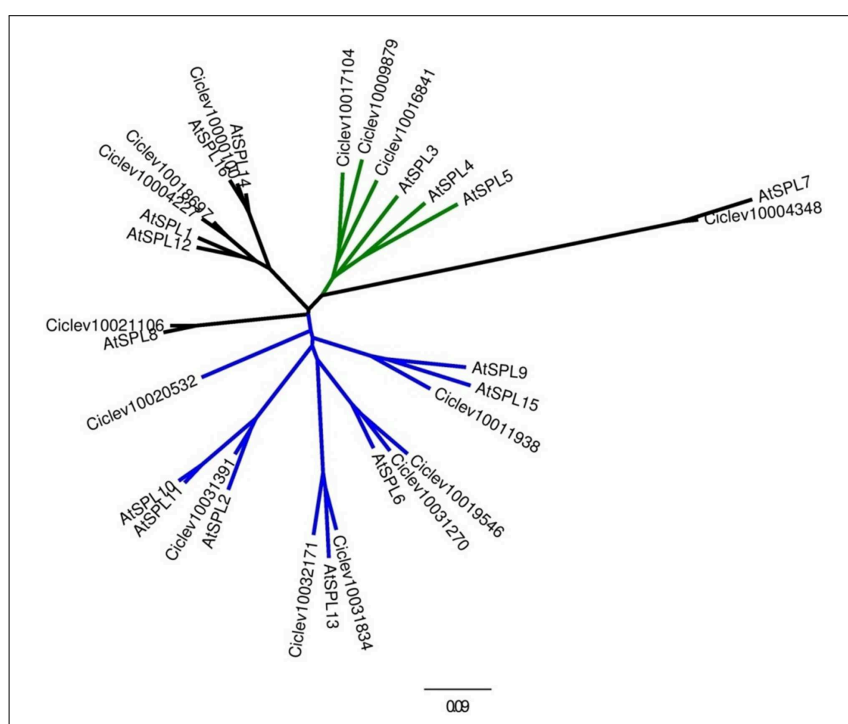

FIGURE 2 | Evolutionary relationships of SPL members from Citrus and Arabidopsis. The maximum likelihood tree is based on the SBP domains (Supplementary Table 3). SPLs were classified into three subgroups: $<200$ amino acids with miR156-binding site located within the $3^{\prime}$ UTR (green), >300 amino acids with miR156-binding site located within the ORF (blue), $>300$ amino acids without miR156 binding site (black).

130, 143 and 189 amino acids, respectively. The second group (blue clade) consisted of SPLs characterized by longer protein sequences ( $>300$ amino acids) and a miR156-binding site located within the ORF. The seven Citrus SPL members within this clade were: Ciclev10020532, Ciclev10031391, Ciclev10032171, Ciclev10031834, Ciclev10031270, Ciclev10019546, and Ciclev10011938. The third group (black clades) consisted of SPLs which did not contain a miR156-binding site. The five Citrus SPL members within this group were: Ciclev10021106, Ciclev10004227, Ciclev10018697, Ciclev10000100, and Ciclev10004348. connecting lines. White boxes represent un-translated regions and gray boxes represent coding regions. The dotted vertical lines indicate the location of the miR156-binding sites; their sequences are shown to the left.

\section{Molecular Characterization of miR156-Regulated CiSPL5}

The roles of SPLs and miR156 as regulators of phase transitions have been extensively studied in Arabidopsis and other annual plant species; however, considerably less research has been done with trees. To gain insight into the roles of SPLs in Citrus and understand whether the presence of a miR156-binding site in an SPL transcript constitutes a real regulatory element, we performed a molecular and functional characterization of one Citrus SPL, Ciclev10009879, whose expression had been previously studied in relation to fruit-load effect on flowering induction of Citrus (Shalom et al., 2012, 2014). Full-length sequencing of Ciclev10009879 mRNA revealed that it is $843 \mathrm{bp}$ long (with one alternative polyadenylation site at $750 \mathrm{bp}$ ) with a putative ORF encoding a 130-amino acid protein and a miR156binding site located in the $3^{\prime}$ UTR. An antisense transcript (AST) of about $2400 \mathrm{bp}$ which encompasses the entire region of Ciclev10009879 was also identified (Figure 3). Surprisingly, RACE analyses indicated that the transcription start site of this AST is located in a neighboring upstream gene, Ciclev10009133, encoding a putative PROTEIN PHOSPHATASE 2C (PP2C). In fact, the full-length structure of the AST was similar to one of the predicted alternative transcripts of Ciclev10009133, only with a longer than predicted $3^{\prime}$ tail (long PP2C transcript, Figure 3). The RACE analyses identified four transcription start sites and four polyadenylation sites in the AST, suggesting a complex mode of transcription. In Citrus, fruit set takes place during May, whereas September-October are regarded as the last time points at which fruit removal during the $\mathrm{ON}$ year reverses the $\mathrm{AB}$ trend (Martinez-Fuentes et al., 2010). The floral induction period starts in mid-November and lasts until approximately mid-January (Davenport, 1990). Expression analysis of the long AST in buds showed that it was expressed at higher levels from May to September and lower levels from November to January (Supplementary Figure 1). However, no significant differences were detected between buds of $\mathrm{ON}$ - and OFF-Crop trees, and no alterations were detected following fruit 


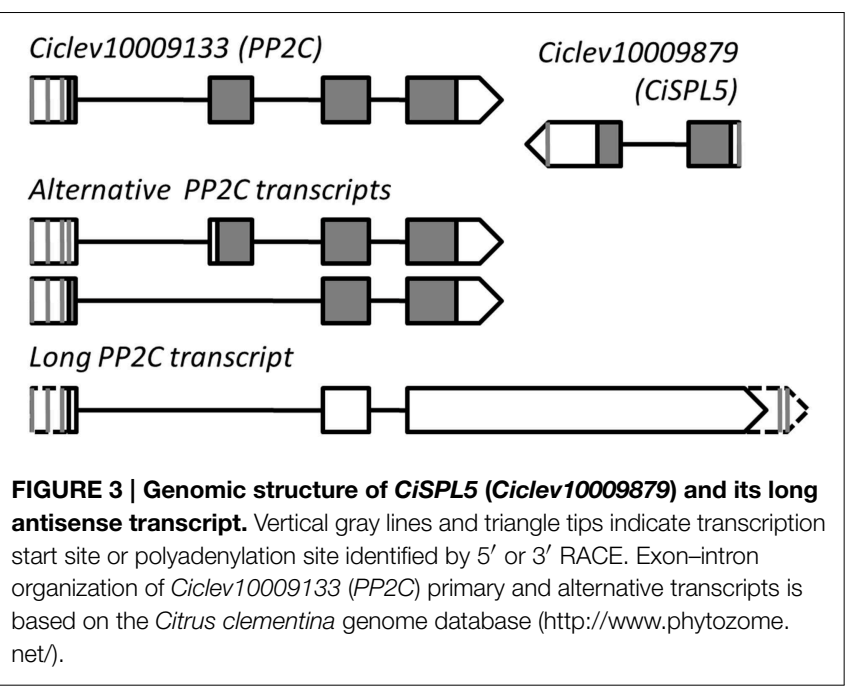

removal, putting its role in flowering control by fruit load into question. As mentioned above, Ciclev10009133 encoded a protein that is highly homologous to Arabidopsis PP2C (At3g15260; $79 \%$ identity). In the Arabidopsis genome, this gene is located jointly and in antisense orientation to AtSPL5, which belongs to the small SPLs subgroup (Figure 2). Therefore, based on SBPdomain sequence homology, protein length, miR156-binding site position and genomic coupling with PP2C, we determined that Citrus SPL Ciclev10009879 is the ortholog of Arabidopsis AtSPL5, and it is henceforth referred to as CiSPL5.

\section{CiSPL5 Promotes Flowering in Arabidopsis and is Repressed by miR156}

Transgenic Arabidopsis plants expressing CiSPL5 under the control of CaMV $35 \mathrm{~S}$ promoter in either $m i R 156$-sensitive (containing the native miR156-binding site) or miR156insensitive forms (not containing the native miR156-binding site) were generated and phenotyped. Three constructs were used: CiSPL5 ORF transcript with its normal 3'UTR (miR156sensitive, 35S::CiSPL5 + 3'UTR, Supplementary Figure 2-1), CiSPL5 ORF transcript lacking the 3'UTR (miR156-insensitive, 35S::CiSPL5 no3'UTR, Supplementary Figure 2-2) and CiSPL5 ORF transcript with 10 mutations in the $3^{\prime}$ UTR miR156-binding site (miR156-insensitive, 35S::CiSPL5 + $m 3^{\prime} U T R$, Supplementary Figure 2-3). Characterization of the CiSPL5-transgenic lines indicated similarity between the 35S::CiSPL5+3'UTR and control (Figure 4A), producing normal size rosette leaves (Figure 4B). The former flowered after $\sim 33$ days under long days (Figure 4C) or $\sim 86$ days under short days (Figure 4D), which was slightly ahead of the control (34 days and 88 days, respectively). In contrast, both 35S::CiSPL5 no3'UTR and $35 S:: C i S P L 5+m 3^{\prime} U T R$ lines produced much smaller and fewer rosette leaves before flowering occurred (Figures 4A,B). Furthermore, time to flowering in these lines was significantly reduced under long days and even more dramatically under short days (Figures 4C,D, respectively). 35S::CiSPL5 no3'UTR and 35S::CiSPL5 $+m 3^{\prime} U T R$ lines had a significantly reduced number of rosette leaves with abaxial trichomes (Figure 4C), characteristic of the adult phase (Telfer et al., 1997), compared to 35S::CiSPL5 + 3'UTR lines and the control lines. Moreover, the formation of abaxial trichomes in 35S::CiSPL5 no3'UTR and 35S::CiSPL5 $+m 3^{\prime} U T R$ lines initiated earlier than in 35S::CiSPL5 + 3'UTR.

\section{Seasonal Expression Patterns of Some CiSPLs and Patterns Following Fruit Removal Coincide with Flowering Induction}

As woody perennials, Citrus trees undergo a series of developmental changes deriving from seasonal environmental and endogenous cues. Some of these changes, such as the shift to flowering and/or vegetation, might be regulated, at least in part, by SPLs. Therefore, we studied their expression patterns in leaves and buds of OFF-Crop trees, expected to flower the following spring, before and during the flowering induction period from November to January. Nine out of fifteen SPLs (Ciclev10020532, Ciclev10021106, Ciclev10031270, Ciclev10031391, Ciclev1000100, Ciclev10019546, Ciclev10032171, Ciclev10031834, and Ciclev10011938) were expressed at higher levels in buds than in leaves at most time points (Figure 5), whereas 4 out of 15 SPLs showed similar expression levels in buds and leaves at most time points. The mRNA levels of one $S P L$, Ciclev10017104, were similar in leaves and buds from May until September, but were higher in leaves from November to January. However, the mRNA of CiSPL5 was exceptional in that at all tested time points, it showed significantly higher levels in leaves than in buds. While CiSPL5 expression in leaves was relatively stable, with a transient threefold increase in November, its expression in buds gradually decreased from May to January (Figure 5, Supplementary Figure 3), consistent with our previous report (Shalom et al., 2012). Similarly, the mRNA levels of Ciclev10020532, Ciclev10021106, and Ciclev10031270 decreased moderately throughout the season in the buds. In contrast, the mRNA levels of Ciclev10019546 in buds showed a moderate increase from May to November followed by a slight decrease in January, similar to the trend in leaves. Another SPL whose expression is worth mentioning is Ciclev10016841, which showed a sharp increase in mRNA levels from November to January.

As heavy fruit load inhibits flowering induction, the mRNA levels of SPLs were also investigated in buds of ON- and OFFCrop trees and following fruit removal (de-fruiting). Most of the SPLs were of similar levels in ON- and OFF-Crop buds, and were not altered significantly by de-fruiting (Figure 6). However, the mRNA levels of Ciclev10020532 and Ciclev10021106 were higher in OFF-Crop vs. ON-Crop buds, and they were significantly upregulated (two- to threefold) 1 week after de-fruiting. In addition, the level of Ciclev10016841 mRNA was significantly higher (by about threefold) in OFF-Crop buds than in ON-Crop buds; however, its level was not altered by de-fruiting.

\section{Fruit Removal Alters the Expression of SPL-related miRNA Genes, but Not Necessarily Abundance of the Mature miRNA}

Primary miRNAs (pri-miRs) are capped and polyadenylated non-coding RNA transcripts containing mature miRNA 


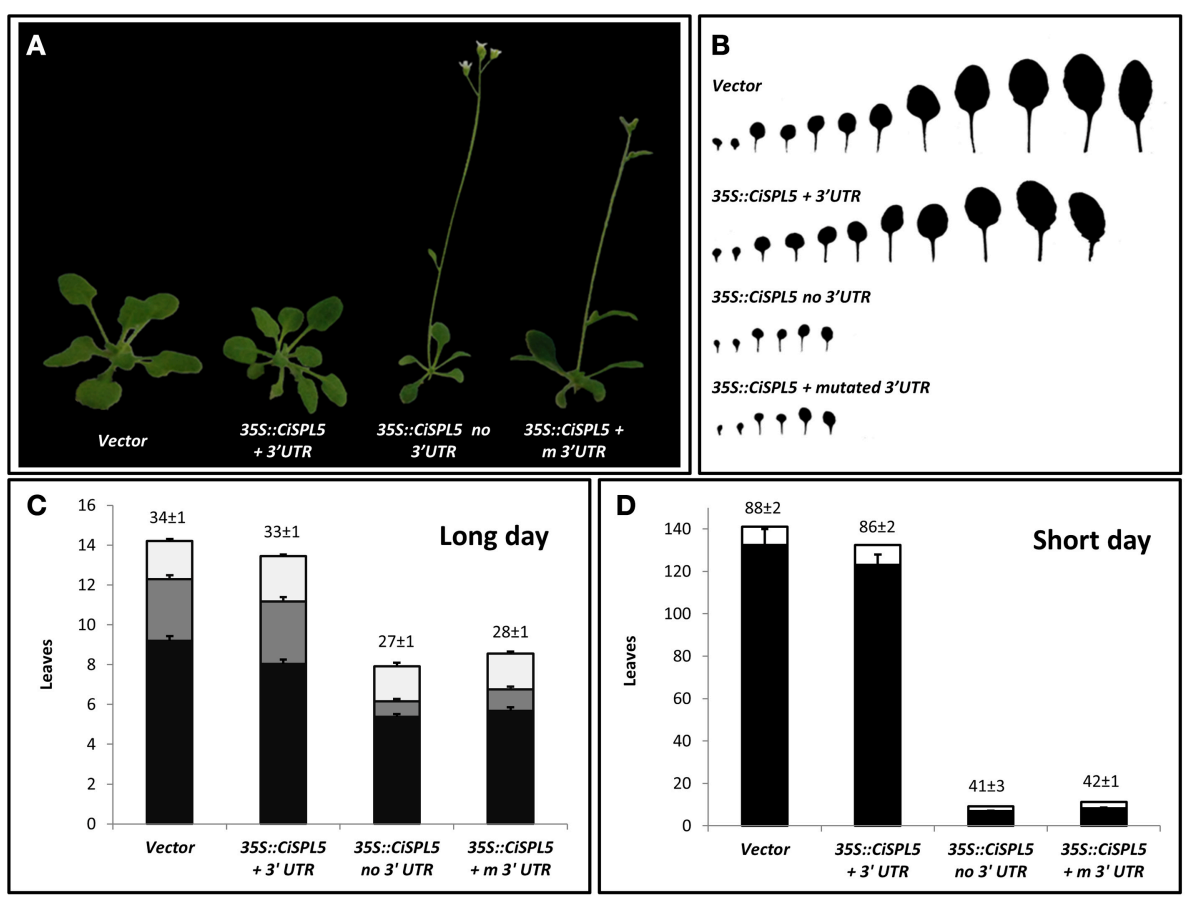

FIGURE 4 | CiSPL5 promotes flowering in Arabidopsis and is repressed by $\boldsymbol{m i R 1 5 6 . ~ ( A ) ~ M o r p h o l o g y ~ o f ~ t r a n s g e n i c ~ p l a n t s ~}$ expressing miR156-sensitive (35S::CiSPL5 + 3'UTR) and miR156-insensitive (35S::CiSPL5 no3'UTR, 35S::CiSPL5 + m3'UTR) versions of CISPL5 under the regulation of the $35 \mathrm{~S}$ promoter. (B) Morphology of rosette leaves in the transgenic plants. (C) The number of leaves without abaxial trichomes (black bars), with abaxial trichomes (gray bars), cauline leaves (white bars) and flowering time (days after planting, top of bar \pm SE) for the transgenic lines under long days. (D) The number of rosette leaves (black bars), cauline leaves (white bars) and flowering time (days after planting, top of bar $\pm \mathrm{SE}$ ) for the transgenic lines under short days. sequences (Voinnet, 2009). Cleavage of the primary miRNA transcript eventually results in the release of biologically active mature miRNA and ultimately, the degradation or translational repression of mRNA targets. The miRBase database (http:// www.mirbase.org/) contains sequence data (precursor and mature) for 75 miRNAs from Citrus. We recently conducted an RNA deep-sequencing analysis of Citrus buds following fruit removal (Shalom et al., 2014). In the current work, we aligned the RNA deep-sequencing data to the Citrus precursor sequences from miRBase. Results of this analysis indicated that about two-thirds of them are expressed in buds; however, only a few were significantly affected by fruit load. Among these were ctr-MIR156 and csi-MIR172a, with the latter expressed to much higher levels (Figure 7A). Expression levels of $c t r$-MIR156 were significantly higher in ON-Crop buds as compared to OFFCrop buds and decreased following de-fruiting (Figure 7B). In contrast, expression levels of csi-MIR172a showed the opposite trend, with OFF-Crop buds and buds after de-fruiting showing higher expression levels than ON-Crop buds. Alterations in both genes occurred as early as 1 week after de-fruiting (Figure 7B). Abundance analysis of the mature miRNA sequences was performed next. While miR156 abundance was not affected by fruit presence, $m i R 172$ was more abundant in OFF-Crop buds than ON-Crop buds, and increased significantly (two to threefold) after de-fruiting (Figure 7C).

\section{Discussion}

\section{SPL Gene Family in Citrus}

Genes containing the SBP domain were originally identified in Antirrhinum majus and were named SQUAMOSA PROMOTER BINDING PROTEIN based on their ability to interact with the promoter of the floral meristem identity gene SQUAMOSA (Klein et al., 1996). Since then, SPLs have been identified and classified in a number of plant species, including Arabidopsis (Rhoades et al., 2002), rice (Xie et al., 2006), tomato (Salinas et al., 2012), and grape (Hou et al., 2013). Similar to other plants, about two-thirds of the Citrus SPLs contain sequences complementary to miR156. In Arabidopsis, 3 of the 10 miR156regulated SPLs, AtSPL3/4/5, differ from the others by the position of their miR156-binding site-it is located in the $3^{\prime}$ UTR and is believed to have moved there via exon degeneration (Guo et al., 2008) - and by their relatively short protein size, which mainly comprises the SBP domain (Wu and Poethig, 2006; Yamaguchi et al., 2009). Overexpression of each of these SPLs shortens the juvenile period and induces early flowering ( $\mathrm{Wu}$ and Poethig, 2006). AtSPL3 activates LEAFY (LFY), FRUITFUL (FUL), and APETALA1 (AP1) by directly binding their promoter regions and evidence suggests that AtSPL4 and AtSPL5 have overlapping functions (Yamaguchi et al., 2009). Our phylogenetic analysis showed that AtSPL3/4/5 have three close orthologs 


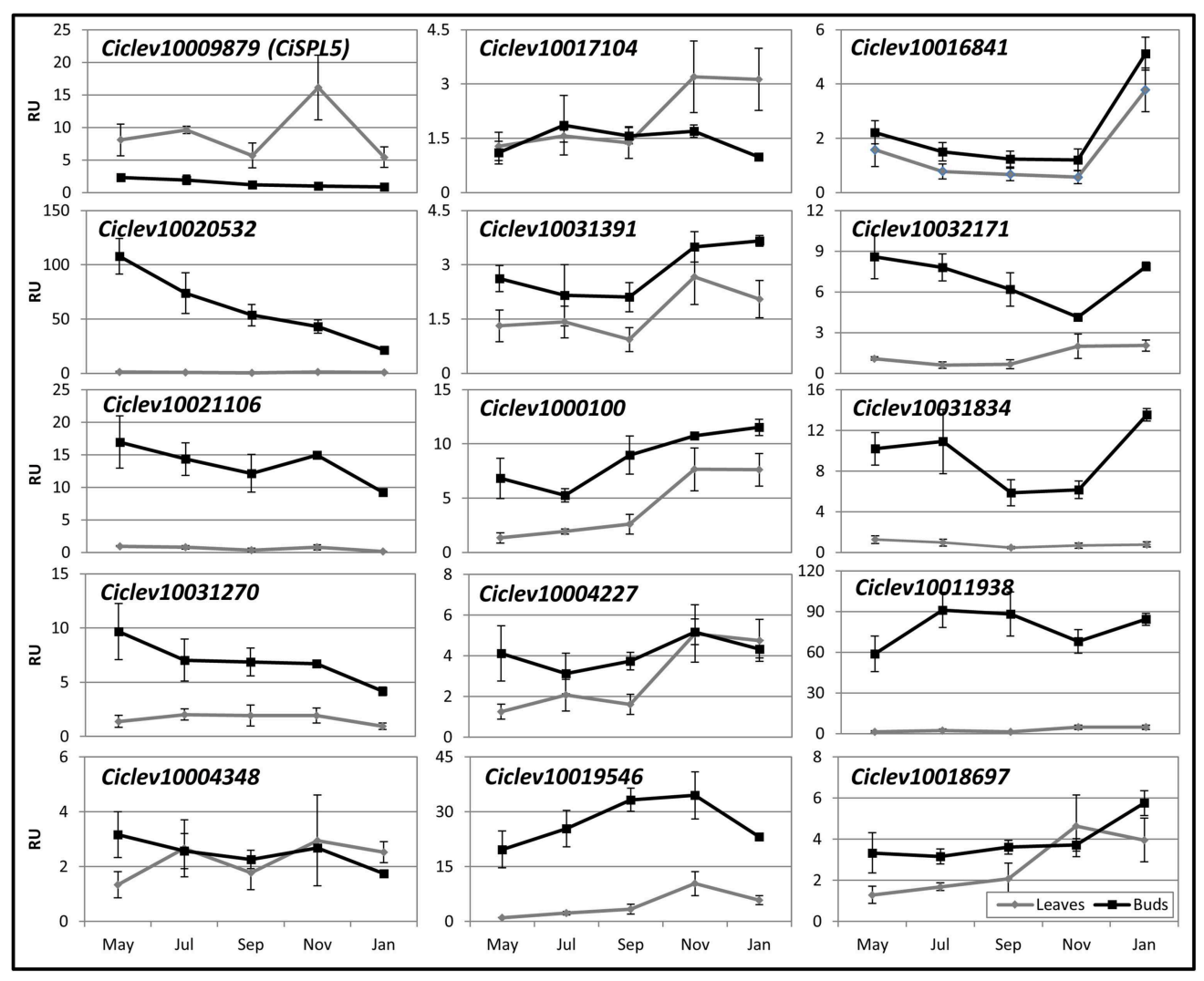

FIGURE 5 | Seasonal expression patterns of Citrus SPLs in OFF-Crop leaves and buds. The mRNA levels (RU, relative units) of the indicated genes were determined in OFF-Crop leaves (gray lines) and buds (black lines) at the indicated months. The numbers are mean values of three independent biological replicates \pm SE. The pattern in the bud is shown in Supplementary Figure $\mathbf{3}$ with extended axis. in Citrus, with similar structural characteristics and therefore potentially similar functions. By its structure and genomic localization (i.e., proximity to a PP2C-like gene), Ciclev10009879 (CiSPL5) is suggested to be the ortholog of Arabidopsis AtSPL5.

\section{Antisense Transcription within CiSPL5}

We identified an AST which encompasses the CiSPL5 genomic region. A large number of overlapping transcripts in antisense orientation with the potential to form double-stranded RNA structures have been identified in the Arabidopsis genome (Jen et al., 2005; Wang et al., 2005), for instance, in AtSPL3 (Wu and Poethig, 2006). Although the role of these ASTs remains unclear, they might function as an additional level of regulation. The identified AST is a long variant of a neighboring gene encoding PP2C-like protein homologs of At3g15260. Some PP2C proteins play a role in abscisic acid (ABA) signaling (Umezawa et al., 2009). ABA homeostasis is affected by fruit load (Shalom et al., 2014), and the decrease in the AST's expression during the winter months somewhat resembles the expression pattern of CiSPL5 (although it does not respond to fruit load). Therefore, exploration of ABA's possible signaling role in CiSPL5 regulation is warranted.

\section{Do SPLs and miR172 Play a Role in Fruit-load Effect on Flowering in Citrus?}

CiSPL5 was able to accelerate Arabidopsis flowering while miR156 repressed its action (Figure 4). Although no direct evidence was provided, it was likely that CiSPL5 regulated phase transition in Citrus, as well. A significant difference in its expression was detected between ON- and OFF-Crop buds, and it was induced by de-fruiting (Shalom et al., 2012, 2014), raising the posebility it also played a role in $\mathrm{AB}$ regulation. However, the findings that the levels of miR156 in Citrus buds were not affected by fruit load (Shalom et al., 2012, Figure 7C), questioned the possibility that fruit load regulated CiSPL5 expression through miR156. Previous investigations in various plant species demonstrated that miR156 was predominant in juvenile tissues, whereas miR172 was induced in adult tissues (Aukerman and Sakai, 2003; Chen, 2004; Lauter et al., 2005; Wu and Poethig, 2006; Chuck et al., 2007). Our transcriptomic data (Figure 7) showed low expression levels of $c t r-M I R 156$ relative to $c s i-M I R 172 a$, which is in agreement with the accepted dogma of their respective roles in juvenile and adult plants. This could explain why the levels of miR156 did not correlate with fruit load. Additional finding was the lack of correlation between the expression patterns of $c t r-$ MIR156 and mature miR156 (Figure 7). It might be that the final 


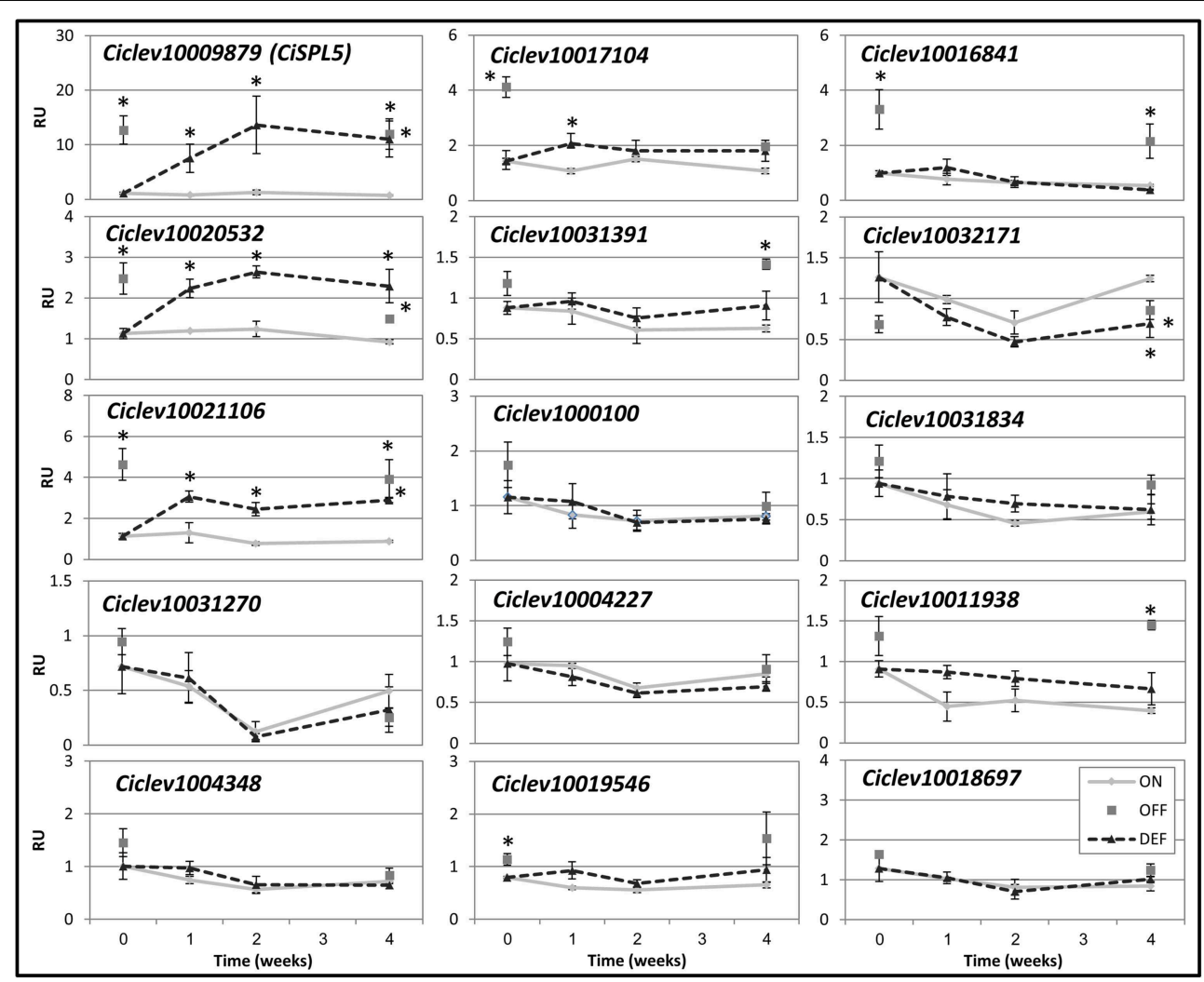

FIGURE 6 | Expression patterns of Citrus SPLs in buds following fruit removal. The mRNA levels ( $R U$, relative units) of the indicated genes were determined in ON-Crop (ON, diamonds), OFF-Crop (OFF, squares), and de-fruited (DEF, dashed lines) trees at the indicated weeks after de-fruiting. The numbers are mean values of three independent biological replicates \pm SE. Asterisks represent significant difference $(P=0.05)$ from ON-Crops at the same time point. CiSPL5 graph was presented in Shalom et al. (2014).
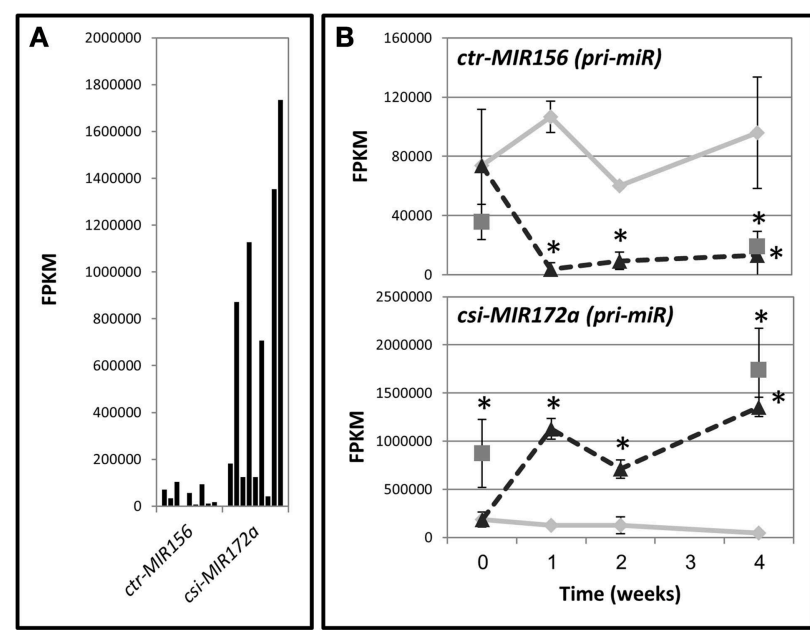

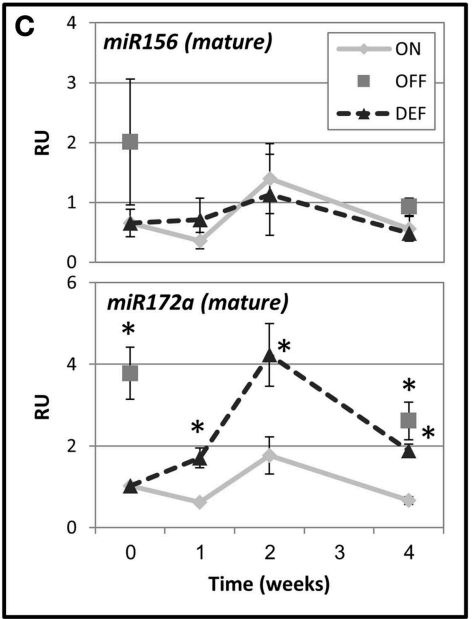

FIGURE 7 | miR156 and miR172 levels in buds following fruit removal. (A) Expression levels of ctr-MIR156 and csi-MIR172a genes are based on transcriptomic data and are represented by FPKM-values, which normalize the read count by the length of the fragment and the total number of mapped reads. (B) Expression patterns of ctr-MIR156 and csi-MIR172a genes after fruit removal. (C) Abundance of the mature
miR156 and miR172a sequences after fruit removal. For (B,C), values were determined in ON-Crop (ON), OFF-Crop (OFF), and de-fruited (DEF) trees at the indicated weeks after de-fruiting. The numbers are mean values of three independent biological replicates \pm SE. Asterisks represent significant difference $(P \leq 0.05)$ from $\mathrm{ON}$ at the same time point. pri-miR, primary mRNA. 
level of this miRNA was ultimately determined by a combination of factors, including additional active miR156 genes or small interfering (si) RNAs.

Unlike miR156, miR172a increased both at the precursor and mature levels following de-fruiting (Figure 7). In Arabidopsis, miR172 transcription is induced by AtSPL9 and AtSPL10 (Wu et al., 2009), and in turn promotes flowering by repressing AP2-like TFs which negatively regulate FT expression (Mathieu et al., 2009). Could this also be the case in Citrus fruit loadaffected flowering? In parallel to $c s i-M I R 172 a$ induction, three CiSPL genes were up-regulated following de-fruiting in the bud (Figure 6): CiSPL5 (Ciclev10009879), Ciclev10020532, and Ciclev10021106. While Ciclev10020532 has no close ortholog in Arabidopsis (Figure 2), Ciclev10021106 is the ortholog of AtSPL8, found to play important roles in the regulation of male fertility (Xing et al., 2010) and gynoecium patterning (Xing et al., 2013). Whether Ciclev10021106 has similar roles in Citrus is unclear; however, it is not targeted by miR156. Taken together, it is tempting to speculate that some of these SPLs regulate miR172a expression by a mechanism similar to that in Arabidopsis, as mentioned above.

\section{miR156's Negative Regulation of CiSPL5 in Arabidopsis Can Be Bypassed through the Photoperiod Pathway}

In addition to flowering promotion, CiSPL5 shortened the juvenile phase, as reflected by positive regulation of abaxial trichome production, which is characteristic of adult leaves. This has also been shown for other SPLs (Cardon et al., 1997; Wang et al., 2009; Wu et al., 2009). While long day promotes Arabidopsis flowering, under short day, flowering occurs after a long period of vegetative growth, as a result of miR156 downregulation accompanied by a gradual increase in AtSPL3 and AtSPL9 expression (Wang et al., 2009). Shifting plants from short days to long days leads to a rapid increase in AtSPL3 and AtSPL9 expression while $m i R 156$ levels remain unchanged. Our functional analysis of CiSPL5 in Arabidopsis supports the notion that the negative regulation by miR156 can be bypassed through

\section{References}

Aukerman, M. J., and Sakai, H. (2003). Regulation of flowering time and floral organ identity by a microRNA and its APETALA2-like target genes. Plant Cell 15, 2730-2741. doi: 10.1105/Tpc.016238

Bechtold, N., Ellis, J., and Pelletier, G. (1993). In-planta agrobacterium-mediated gene transfer by infiltration of adult Arabidopsis thaliana plants. C. R. Acad. Sci. 316, 1194-1199.

Cardon, G. H., Hohmann, S., Nettesheim, K., Saedler, H., and Huijser, P. (1997). Functional analysis of the Arabidopsis thaliana SBP-box gene SPL3: a novel gene involved in the floral transition. Plant J. 12, 367-377. doi: 10.1046/j.1365313X.1997.12020367.x

Chang, S., Puryear, J., and Cairney, J. (1993). A simple and efficient method for isolating RNA from pine trees. Plant Mol. Biol. Rep. 11, 113-116.

Chen, X. M. (2004). A microRNA as a translational repressor of APETALA2 in Arabidopsis flower development. Science 303, 2022-2025. doi: $10.1126 /$ science. 1088060

Chuck, G., Cigan, A. M., Saeteurn, K., and Hake, S. (2007). The heterochronic maize mutant Corngrass1 results from overexpression of a tandem microRNA. Nat. Genet. 39, 544-549. doi: 10.1038/ $\operatorname{Ng} 2001$ the photoperiod pathway; when overexpressed in miR156insensitive forms, CiSPL5 accelerated flowering regardless of day length. The normal phenotype of lines overexpressing CiSPL5 in a $m i R 156$-sensitive form indicated that flowering in these plants is affected by endogenous factors, such as an age-dependent pathway (short day) or the photoperiod pathway (long day).

\section{Funding}

This work was supported by fund number 203-0870 of the Chief Scientist of the Ministry of Agriculture and Rural Development.

\section{Supplementary Material}

The Supplementary Material for this article can be found online at: http://journal.frontiersin.org/article/10.3389/fpls.2015. 00389/abstract

Supplementary Figure 1 | Expression of CiSPL5 antisense transcript (long PP2C transcript) in buds. The mRNA levels (RU, relative units) of the long PP2C transcript were determined in ON-Crop (ON) and OFF-Crop (OFF) buds at the indicated months (upper graph) and in ON-Crop (ON) and de-fruited (DEF) trees at the indicated weeks after de-fruiting (lower graph) by nCounter analysis (described in Shalom et al., 2014). The numbers are mean values of three independent biological replicates $\pm \mathrm{SE}$.

Supplementary Figure 2 | Constructs used for constitutive expression of CiSPL5 with different modifications. The sequences of the native or mutated miR156 target sites are illustrated. The native site is indicated by a dotted black line and a mutated site is indicated by a dotted gray line.

\section{Supplementary Figure 3 | Seasonal expression pattern of CiSPL5 in}

OFF-Crop buds. The mRNA levels (RU, relative units) of CiSPL5 were determined in OFF-Crop buds at the indicated months. The numbers are mean values of three independent biological replicates \pm SE.

Supplementary Table1 | List of primers used in this study.

Supplementary Table2 | Precursor sequences of ctr-MIR156 and csi-MIR172a from miRBase (http://www.mirbase.org/).

Supplementary Table3 | SBP-domain sequences of all 15 SPLs from Citrus.

Supplementary Table4 | RACE primers specific for Citrus SPL.

Davenport, T. L. (1990). Citrus flowering. Horticul Rev. 12, 349-408. doi: 10.1002/9781118060858.ch8

Edgar, R. C. (2004). MUSCLE: multiple sequence alignment with high accuracy and high throughput. Nucleic Acids Res. 32, 1792-1797. doi: 10.1093/Nar/Gkh340

Gleave, A. P. (1992). A versatile binary vector system with a T-DNA organizationalstructure conducive to efficient integration of cloned DNA into the plant genome. Plant Mol. Biol. 20, 1203-1207. doi: 10.1007/Bf00028910

Gou, J. Y., Felippes, F. F., Liu, C. J., Weigel, D., and Wang, J. W. (2011). Negative regulation of anthocyanin biosynthesis in arabidopsis by a miR156-targeted SPL transcription factor. Plant Cell 23, 1512-1522. doi: 10.1105/tpc.111.084525

Guindon, S., and Gascuel, O. (2003). A simple, fast, and accurate algorithm to estimate large phylogenies by maximum likelihood. Syst. Biol. 52, 696-704. doi: 10.1080/10635150390235520

Guo, A. Y., Zhu, Q. H., Gu, X. C., Ge, S., Yang, J., and Luo, J. C. (2008). Genomewide identification and evolutionary analysis of the plant specific SBP-box transcription factor family. Gene 418, 1-8. doi: 10.1016/j.gene.2008.03.016

Haas, B. J., Papanicolaou, A., Yassour, M., Grabherr, M., Blood, P. D., Bowden, J., et al. (2013). De novo transcript sequence reconstruction from RNA-seq using the Trinity platform for reference generation and analysis. Nat. Protoc. 8, 1494-1512. doi: 10.1038/nprot.2013.084 
Hou, H. M., Li, J., Gao, M., Singer, S. D., Wang, H., Mao, L. Y., et al. (2013). Genomic organization, phylogenetic comparison and differential expression of the SBP-box family genes in grape. PLoS ONE 8:e59358. doi: 10.1371/journal.pone.0059358

Jen, C. H., Michalopoulos, I., Westhead, D. R., and Meyer, P. (2005). Natural antisense transcripts with coding capacity in Arabidopsis may have a regulatory role that is not linked to double-stranded RNA degradation. Genome Biol. 6:R51. doi: 10.1186/Gb-2005-6-6-R51

Jung, J. H., Seo, Y. H., Seo, P. J., Reyes, J. L., Yun, J., Chua, N. H., et al. (2007). The GIGANTEA-regulated microRNA172 mediates photoperiodic flowering independent of CONSTANS in Arabidopsis. Plant Cell 19, 2736-2748. doi: 10.1105/tpc.107.054528

Kim, J. J., Lee, J. H., Kim, W., Jung, H. S., Huijser, P., and Ahn, J. H. (2012). The microRNA156-SQUAMOSA PROMOTER BINDING PROTEINLIKE3 module regulates ambient temperature-responsive flowering via FLOWERING LOCUS T in Arabidopsis. Plant Physiol. 159, 461-478. doi: 10.1104/pp.111.192369

Klein, J., Saedler, H., and Huijser, P. (1996). A new family of DNA binding proteins includes putative transcriptional regulators of the Antirrhinum majus floral meristem identity gene SQUAMOSA. Mol. Gen. Genet. 250, 7-16. doi: 10.1007/Bf02191820

Langmead, B., Trapnell, C., Pop, M., and Salzberg, S. L. (2009). Ultrafast and memory-efficient alignment of short DNA sequences to the human genome. Genome Biol. 10:R25. doi: 10.1186/Gb-2009-10-3-R25

Lauter, N., Kampani, A., Carlson, S., Goebel, M., and Moose, S. P. (2005). microRNA172 down-regulates glossy15 to promote vegetative phase change in maize. Proc. Natl. Acad. Sci. U.S.A. 102, 9412-9417. doi: 10.1073/pnas.0503927102

Li, B., and Dewey, C. N. (2011). RSEM: accurate transcript quantification from RNA-seq data with or without a refference genome. BMC Bioinformatics 12:323. doi: 10.1186/1471-2105-12-323

Martinez-Fuentes, A., Mesejo, C., Reig, C., and Agusti, M. (2010). Timing of the inhibitory effect of fruit on return bloom of 'Valencia' sweet orange (Citrus sinesis (L.) Osbek). J. Sci. Food Agric. 90, 1936-1943. doi: 10.1002/jsfa.4038

Mathieu, J., Yant, L. J., Murdter, F., Kuttner, F., and Schmid, M. (2009). Repression of flowering by the miR172 target SMZ. PLoS Biol. 7:e1000148. doi: 10.1371/journal.pbio. 1000148

Monselise, S. P., and Goldschmidt, E. E. (1982). Alternate bearing in fruit trees. Hortic. Rev. 4, 128-173.

Rhoades, M. W., Reinhart, B. J., Lim, L. P., Burge, C. B., Bartel, B., and Bartel, D. P. (2002). Prediction of plant microRNA targets. Cell 110, 513-520. doi: 10.1016/S0092-8674(02)00863-2

Robinson, M. D., and Oshlack, A. (2010). A scaling normalization method for differential expression analysis of RNA-seq data. Genome Biol. 11:R25. doi: 10.1186/Gb-2010-11-3-R25

Salinas, M., Xing, S. P., Hohmann, S., Berndtgen, R., and Huijser, P. (2012). Genomic organization, phylogenetic comparison and differential expression of the SBP-box family of transcription factors in tomato. Planta 235, 1171-1184. doi: 10.1007/s00425-011-1565-y

Shalom, L., Samuels, S., Zur, N., Shlizerman, L., Doron-Faigenboim, A., Blumwald, E., et al. (2014). Fruit load induces changes in global gene expression and in abscisic acid (ABA) and indole acetic acid (IAA) homeostasis in citrus buds. J. Exp. Bot. 65, 3029-3044. doi: 10.1093/Jxb/Eru148

Shalom, L., Samuels, S., Zur, N., Shlizerman, L., Zemach, H., Weissberg, M., et al. (2012). Alternate bearing in citrus: changes in the expression of flowering control genes and in global gene expression in ON-versus OFF-crop trees. PLoS ONE 7:e46930. doi: 10.1371/journal.pone.0046930

Song, C., Fang, J., Li, X., Liu, H., and Chao, C. T. (2009). Identification and charactarization of 27 conserved microRNAs in citrus. Planta 230, 671-685. doi: 10.1007/s00425-009-0971-x

Telfer, A., Bollman, K. M., and Poethig, R. S. (1997). Phase change and the regulation of trichome distribution in Arabidopsis thaliana. Development 124, 645-654

Umezawa, T., Sugiyama, N., Mizoguchi, M., Hayashi, S., Myouga, F., YamaguchiShinozaki, K., et al. (2009). Type 2C protein phosphatases directly regulate abscisic acid-activated protein kinases in Arabidopsis. Proc. Natl. Acad. Sci. U.S.A. 106, 17588-17593. doi: 10.1073/pnas.0907095106

Unte, U. S., Sorensen, A. M., Pesaresi, P., Gandikota, M., Leister, D., Saedler, H., et al. (2003). SPL8, an SBP-Box gene that affects pollen sac development in Arabidopsis. Plant Cell 15, 1009-1019. doi: 10.1105/Tpc. 010678

Usami, T., Horiguchi, G., Yano, S., and Tsukaya, H. (2009). The more and smaller cells mutants of Arabidopsis thaliana identify novel roles for SQUAMOSA PROMOTER BINDING PROTEIN-LIKE genes in the control of heteroblasty. Development 136, 955-964. doi: 10.1242/Dev.028613

Voinnet, O. (2009). Origin, Biogenesis, and activity of plant microRNAs. Cell 136, 669-687. doi: 10.1016/j.cell.2009.01.046

Wahl, V., Ponnu, J., Schlereth, A., Arrivault, S., Langenecker, T., Franke, A., et al. (2013). Regulation of flowering by trehalose-6-phosphate signaling in Arabidopsis thaliana. Science 339, 704-707. doi: 10.1126/science.1230406

Wang, J. W., Czech, B., and Weigel, D. (2009). miR156-regulated SPL transcription factors define an endogenous flowering pathway in Arabidopsis thaliana. Cell 138, 738-749. doi: 10.1016/j.cell.2009.06.014

Wang, J. W., Park, M. Y., Wang, L. J., Koo, Y. J., Chen, X. Y., Weigel, D., et al. (2011). MiRNA control of vegetative phase change in trees. PLoS Genet. 7:e1002012. doi: 10.1371/journal.pgen.1002012

Wang, X. J., Gaasterland, T., and Chua, N. H. (2005). Genome-wide prediction and identification of cis-natural antisense transcripts in Arabidopsis thaliana. Genome Biol. 6:R30. doi: 10.1186/Gb-2005-6-4-R30

Wu, G., Park, M. Y., Conway, S. R., Wang, J. W., Weigel, D., and Poethig, R. S. (2009). The sequential action of miR156 and miR172 regulates developmental timing in Arabidopsis. Cell 138, 750-759. doi: 10.1016/j.cell.2009.06.031

Wu, G., and Poethig, R. S. (2006). Temporal regulation of shoot development in Arabidopsis thaliana by miR156 and its target SPL3. Development 133, 3539-3547. doi: 10.1242/Dev.02521

Xie, K. B., Wu, C. Q., and Xiong, L. Z. (2006). Genomic organization, differential expression, and interaction of SQUAMOSA promoter-binding-like transcription factors and microRNA156 in rice. Plant Physiol. 142, 280-293. doi: $10.1104 /$ pp.106.084475

Xing, S. P., Salinas, M., Garcia-Molina, A., Hohmann, S., Berndtgen, R., and Huijser, P. (2013). SPL8 and miR156-targeted SPL genes redundantly regulate Arabidopsis gynoecium differential patterning. Plant J. 75, 566-577. doi: 10.1111/Tpj.12221

Xing, S. P., Salinas, M., Hohmann, S., Berndtgen, R., and Huijser, P. (2010). miR156-targeted and nontargeted SBP-box transcription factors act in concert to secure male fertility in Arabidopsis. Plant Cell 22, 3935-3950. doi: $10.1105 /$ tpc. 110.079343

Xu, Q., Liu, Y. L., Zhu, A. D., Wu, X. M., Ye, J. L., Yu, K. Q., et al. (2010). Discovery and comparative profiling of microRNAs in a sweet orange red-flesh mutant and its wild type. BMC Genomics 11:246. doi: 10.1186/1471-2164-11-246

Yamaguchi, A., Wu, M. F., Yang, L., Wu, G., Poethig, R. S., and Wagner, D. (2009). The microRNA-regulated SBP-box transcription factor SPL3 is a direct upstream activator of LEAFY, FRUITFULL, and APETALA1. Dev. Cell 17, 268-278. doi: 10.1016/j.devcel.2009.06.007

Yamasaki, H., Hayashi, M., Fukazawa, M., Kobayashi, Y., and Shikanai, T. (2009). SQUAMOSA Promoter Binding Protein-Like7 is a central regulator for copper homeostasis in Arabidopsis. Plant Cell 21, 347-361. doi: 10.1105/tpc.108.060137

Yu, N., Cai, W. J., Wang, S. C., Shan, C. M., Wang, L. J., and Chen, X. Y. (2010). Temporal control of trichome distribution by microRNA156targeted SPL genes in Arabidopsis thaliana. Plant Cell 22, 2322-2335. doi: $10.1105 /$ tpc.109.072579

Yu, S., Galvao, V. C., Zhang, Y. C., Horrer, D., Zhang, T. Q., Hao, Y. H., et al. (2012). Gibberellin regulates the arabidopsis floral transition through miR156 targeted SQUAMOSA PROMOTER BINDING-LIKE transcription factors. Plant Cell 24, 3320-3332. doi: 10.1105/tpc.112.101014

Conflict of Interest Statement: The authors declare that the research was conducted in the absence of any commercial or financial relationships that could be construed as a potential conflict of interest.

Copyright (c) 2015 Shalom, Shlizerman, Zur, Doron-Faigenboim, Blumwald and Sadka. This is an open-access article distributed under the terms of the Creative Commons Attribution License (CC BY). The use, distribution or reproduction in other forums is permitted, provided the original author(s) or licensor are credited and that the original publication in this journal is cited, in accordance with accepted academic practice. No use, distribution or reproduction is permitted which does not comply with these terms. 